\title{
EFICÁCIA DE DIFERENTES FÁRMACOS NO CONTROLE PARASITÁRIO EM OVINOS
}

\author{
OLIVEIRA, Plínio Aguiar ${ }^{1}$, \\ PINTO, Diego Moscarelli ${ }^{2}$, \\ RUAS, Jerônimo Lopes ${ }^{3}$, \\ SANTOS, Tânia Regina Bettin dos ${ }^{2}$, \\ PAPPEN, Felipe Geraldo ${ }^{4}$, \\ SALVADEGO, Taís Aparecida ${ }^{5}$, \\ BORBA, Tailana Cristina de ${ }^{5}$, \\ FERIGOLLO, Ana Paula ${ }^{5}$,
}

\footnotetext{
${ }^{1}$ Programa de Pós-Graduação em Parasitologia/UFPEL; ${ }^{2}$ Professor da Faculdade de Veterinária/UFPEL; ${ }^{3}$ Médico Veterinário da Faculdade de Veterinária LRD/UFPEL; ${ }^{4}$ Professor do Instituto Federal Catarinense/IFC Concórdia; ${ }^{5}$ Graduando do Curso de Medicina Veterinária do Instituto Federal Catarinense/IFC - Concórdia.
}

\section{RESUMO}

A s parasitoses intestinais são um grande problema na ovinocultura, pois levam os produtores a utilizar anti-helmínticos em excesso, contribuindo para o desenvolvimento da resistência. Este trabalho objetivou avaliar a eficácia antihelmíntica de diferentes princípios ativos no controle de nematódeos gastrintestinais de um rebanho ovino do Alto Uruguai Catarinense, Oeste de Santa Catarina. Foram utilizadas 82 fêmeas ovinas da raça Texel distribuídas em dois grupos controle e outros seis para testar os seguintes fármacos: closantel, triclorfon, albendazol, disofenol, levamisol, moxidectina, ivermectina e ivermectina associada ao clorsulon. Todos os produtos foram administrados de acordo com as indicações dos respectivos fabricantes. A eficácia foi avaliada pelo cálculo do percentual de redução de ovos por grama de fezes (OPG), realizado a partir dos resultados pré e pós-tratamentos (sete e quatorze dias). As amostras fecais pré-tratamento foram submetidas à coprocultura para identificação dos gêneros de parasitos presentes no rebanho. Sete, dos oito fármacos testados, se mostraram ineficazes para o tratamento das helmintoses. Os percentuais de redução observados aos sete e quatorze dias póstratamento foram, respectivamente, $54,7 \%$ e $84,3 \%$ para o closantel; $93,1 \%$ e $90,4 \%$ para o triclorfon; $0 \%$ em ambas para o albendazole; $100 \%$ e $100 \%$ para o disofenol; $8,4 \%$ e $0 \%$ para - levamisol; e para moxidectina, ivermectina e ivermectina associada ao clorsulon este percentual foi zero. Os nematódeos presentes no rebanho foram Trichostrongylus (20\%) e Haemonchus (80\%). Conclui-se que a maioria dos fármacos disponíveis comercialmente não foi eficaz no tratamento anti-helmíntico, o que demonstra a necessidade de aprimorar opções de combate aos parasitos que não se baseiem exclusivamente no uso de químicos. Estudos complementares são necessários para dimensionar a resistência anti-helmíntica no Oeste de Santa Catarina.

Palavras-chave: Ovinocultura. Anti-helmínticos. Resistência. Verminose. 


\section{INTRODUÇÃO}

O Brasil tem extensão territorial e clima favorável que proporcionam ao país elevado potencial como produtor mundial na ovinocultura. No entanto, as infecções por parasitos internos têm sido o maior problema sanitário enfrentado pelos criadores (RAMOS et al., 2002). Já foi estimado um gasto anual de dois milhões de dólares com anti-helmínticos (ECHEVARRIA et al., 1988).

As maiores perdas ocorrem quando os ovinos são criados em pequenas áreas, com superlotação, proporcionando alta contaminação da pastagem com larvas de terceiro estádio, as quais são fonte de infecção para os animais (SOTOMAIOR et al., 2007).

O constante desafio dos ovinos pelo parasitismo leva os produtores a realizarem vários tratamentos anti-helmínticos ao longo do ano, o que, inevitavelmente, favorece o desenvolvimento de resistência ao grupo químico mais utilizado (AMARANTE et al., 1992; TORRES-ACOSTA et al., 2012). Em alguns casos, a resistência é considerada múltipla, quando presente a mais de um princípio ativo (KAPLAN et al. 2004).

Considera-se que a ocorrência de resistência anti-helmíntica nas propriedades é hoje uma regra e não mais exceção nos países da América Latina (NARI, 2011). No Planalto Sul e Litoral do estado de Santa Catarina, existem relatos de resistência a diferentes moléculas antihelmínticas desde a década passada (RAMOS, et al., 2002).

Baseado no exposto acima, o presente trabalho objetivou avaliar a eficácia anti-helmíntica de diferentes princípios ativos no controle de nematódeos gastrintestinais de um rebanho ovino do Alto Uruguai Catarinense, região Oeste de Santa Catarina.

\section{MATERIAL E MÉTODOS}

\section{Local e Período de Execução}

O trabalho foi realizado no município de Concórdia, Oeste de Santa Catarina, na localização geográfica $27^{\circ} 12^{\prime} 22^{\prime \prime} \mathrm{S}$ e $52^{\circ} 05^{\prime} 22^{\prime \prime} \mathrm{W}$, em altitude de 584 metros. Os exames de fezes foram realizados nos Laboratórios de Parasitologia do Curso de Medicina Veterinária do Instituto 
Federal Catarinense - Campus Concórdia e do Instituto de Biologia da Universidade Federal de Pelotas.

O experimento foi realizado durante os meses de maio e junho de 2010.

\section{Fármacos Testados}

Foram testados oito fármacos distintos, mediante a utilização de produtos comerciais disponíveis no mercado da região: closantel, triclorfon, albendazol, disofenol, levamisol, moxidectina, ivermectina e ivermectina associada ao clorsulon. Todos os produtos foram utilizados de acordo com as recomendações dos fabricantes.

\section{Grupos Experimentais}

Foram utilizadas 82 fêmeas da raça Texel, sendo 33 fêmeas não gestantes, de um ano de idade e 49 fêmeas adultas, possivelmente gestantes. Não foi possível confirmar o diagnóstico de gestação, pois o período reprodutivo da propriedade encerrou sete dias antes do início do experimento. Os animais foram distribuídos ao acaso nos grupos experimentais, considerando-se a possível gestação como fator discriminatório devido à contraindicação de alguns produtos para uso em fêmeas gestantes (Tabela 1). 
Tabela 1 - Composição dos grupos experimentais de acordo com os fármacos testados, faixa etária e estado reprodutivo dos animais.

\begin{tabular}{c|c|c|c|c|c}
\hline Grupo & Dosagem & $\begin{array}{c}\text { Via de } \\
\text { Aplicação }\end{array}$ & $\mathbf{N}$ & Faixa etária & $\begin{array}{c}\text { Estado reprodutivo } \\
\text { estimado*** }\end{array}$ \\
\hline Controle 1 & - & - & 9 & Cordeiras & não gestantes \\
\hline Closantel* & $10 \mathrm{mg} / \mathrm{kg}$ & Oral & 8 & Cordeiras & não gestante \\
\hline Triclorfon* & $97 \mathrm{mg} / \mathrm{kg}$ & Oral & 8 & Cordeiras & não gestantes \\
\hline Albendazol* & $5 \mathrm{mg} / \mathrm{kg}$ & Oral & 8 & Cordeiras & não gestantes \\
\hline Controle 2 & - & - & 9 & Ovelhas & Possivelmente gestantes \\
\hline Disofenol** & $7,5 \mathrm{mg} / \mathrm{kg}$ & Subcutânea & 8 & Ovelhas & Possivelmente gestantes \\
\hline Levamisol** & $4,7 \mathrm{mg} / \mathrm{kg}$ & Subcutânea & 8 & Ovelhas & Possivelmente gestantes \\
\hline Moxidectina** & $0,2 \mathrm{mg} / \mathrm{kg}$ & Subcutânea & 8 & Ovelhas & Possivelmente gestantes \\
\hline Ivermectina** & $0,2 \mathrm{mg} / \mathrm{kg}$ & Subcutânea & 8 & Ovelhas & Possivelmente gestantes \\
\hline $\begin{array}{c}\text { Ivermectina }+ \\
\text { Clorsulon** }\end{array}$ & $\begin{array}{c}0,2 \mathrm{mg} / \mathrm{kg} \\
+2 \mathrm{mg} / \mathrm{Kg}\end{array}$ & Subcutânea & 8 & Ovelhas & Possivelmente gestantes \\
\hline
\end{tabular}

*Anti-helmínticos contra-indicados para fêmeas gestantes;

**Anti-helmínticos sem contra-indicação para fêmeas gestantes;

Nenhum animal recebeu tratamento anti-helmíntico durante os 60 dias que antecederam o início do experimento.

Todos os grupos, inclusive os controles, foram mantidos sob as mesmas condições de manejo ao longo do experimento.

\section{Exames Laboratoriais}

Os exames laboratoriais realizados foram: Técnica de Gordon e Whitlock modificada (1939), para quantificar a presença de ovos de helmintos por grama de fezes (OPG); e Técnica de Roberts e O'Sullivan (1950) para identificar larvas dos respectivos gêneros de nematódeos. 


\section{Percentual de redução de OPG}

O cálculo do percentual de redução foi realizado em cada grupo, a partir do resultado médio da contagem de OPG no dia zero, sete e quatorze dias após o tratamento anti-helmíntico, de acordo com a fórmula proposta por Boersema e Pandey (1997):

$$
P R=\left[\begin{array}{ccc}
1-\left(T_{2} / T_{1}\right) & x\left(C_{1} / C_{2}\right)
\end{array}\right] \times 100
$$

Onde:

PR = Percentual de Redução;

$\mathrm{T}_{1}=\mathrm{OPG}$ médio do grupo antes do tratamento;

$\mathrm{T}_{2}=$ OPG médio do grupo após o tratamento;

$\mathrm{C}_{1}=\mathrm{OPG}$ médio do grupo controle no início do experimento;

$\mathrm{C}_{2}=\mathrm{OPG}$ médio do grupo controle ao final do experimento.

Foram considerados eficazes os fármacos cujos respectivos grupos experimentais apresentaram redução de OPG superior a 95\% (COLES; ROUSH, 1992; WOOD et al., 1995).

\section{RESULTADOS E DISCUSSÃO}

Os resultados da redução da contagem de OPG demonstraram que sete dos oito fármacos testados $(87,5 \%)$ não foram eficazes no tratamento anti-helmíntico dos grupos experimentais (Tabela 2). Estes resultados indicam que a população de nematódeos gastrintestinais presente no rebanho estudado apresenta elevado grau de resistência aos produtos testados já que, na maioria das vezes, os percentuais de redução da contagem de OPG foram inferiores a 95\%. 
Tabela 2 - Resultados percentuais da redução da contagem de OPG aos sete e aos 14 dias de acordo com os grupos experimentais.

\begin{tabular}{cccccc}
\hline Grupo & $\begin{array}{c}\text { Média OPG } \\
\text { Dia Zero }\end{array}$ & $\begin{array}{c}\text { Média OPG } \\
\text { Dia 7 }\end{array}$ & $\begin{array}{c}\text { Média OPG } \\
\text { Dia 14 }\end{array}$ & $\begin{array}{c}\text { \% Redução } \\
\text { Dia 7 }\end{array}$ & $\begin{array}{c}\text { \% Redução } \\
\text { Dia 14 }\end{array}$ \\
\hline Controle 1 & 3180 & 1730 & 3100 & - & - \\
\hline Triclorfontel & 4470 & 1100 & 680 & 54,7 & 84,3 \\
\hline Albendazol & 4810 & 180 & 450 & 93,1 & 90,4 \\
\hline Controle 2 & 2300 & 2000 & 6830 & 0 & 0 \\
\hline Disofenol & 800 & 5070 & 7220 & - & 100 \\
\hline Levamisol & 5260 & 1030 & 4300 & 8,4 & 0 \\
\hline Moxidectina & 3400 & 3150 & 1200 & 0 & 0 \\
\hline Ivermectina & 4160 & 1900 & 8130 & 0 & 0 \\
\hline $\begin{array}{c}\text { Ivermectina } \\
\text { Clorsulon }\end{array}$ & 360 & 3860 & 1600 & 0 & 0 \\
\hline
\end{tabular}

Dos produtos com indicação para fêmeas gestantes, apenas disofenol apresentou eficácia acima do valor mínimo esperado. Também foi o único de todos os grupos testados a apresentar $100 \%$ de redução de contagem de OPG, aos sete e aos quatorze dias, ainda que este seja considerado um anti-helmíntico de pequeno espectro (ALMEIDA; AYRES, 1996).

Produtos a base de levamisol ou avermectinas (moxidectina e ivermectina), mostraram-se ineficazes na redução da contagem de OPG neste estudo. Os primeiros relatos de resistência na década de 90 já destacavam a ineficácia dessas bases químicas em algumas situações (CUNHA FILHO; YAMAMURA, 1999), tendência que tem sido mantida nos últimos anos (NARI, 2011; RAMOS et al., 2002; THOMAZ-SOCCOL et al., 2004; VERÍSSIMO et al., 2012). 
O uso frequente de anti-helmínticos contribui para a seleção de nematódeos capazes de sobreviver aos tratamentos, uma vez que acelera a formação das próximas gerações de parasitos resistentes (COLES, 2005).

Ainda neste sentido, o uso de disofenol, atualmente disponível apenas para administração subcutânea, diminuiu nas propriedades de criação de ovinos lanados pela dificuldade de aplicação por esta via, o que pode ter preservado o potencial antiparasitário deste fármaco.

Os dados do presente estudo são também semelhantes aos encontrados na literatura em testes de redução para produtos à base de albendazol (BOERSEMA; PANDEY, 1997; CUNHA FILHO; YAMAMURA, 1999; RAMOS et al., 2002; THOMAZ-SOCCOL et al., 2004) e closantel (FALBO et al., 2009), o que comprova a ampla resistência dos parasitos aos mesmos.

Já para triclorfon, os resultados de ineficácia aos sete e quatorze dias pós-tratamento (93,1 e 90,4\%), diferem do relatado por FALBO et al. (2009), que constatou eficácia de 97,9\% para este princípio ativo.

Entretanto, se o parâmetro de redução mínima de contagem de OPG adotado neste estudo fosse de 90\%, assim como o proposto por Veríssimo et al. (2012), triclorfon seria o único fármaco classificado até então como ineficaz que poderia ser utilizado no controle parasitário deste rebanho. Este resultado, acima da média dos demais, está provavelmente ligado ao fato de que sua formulação comercial, em pó, exige maior tempo do proprietário para realizar a diluição no momento do uso, e por isso acaba sendo menos empregado no dia a dia. Além disso, o grupamento dos organofosforados possui alta toxicidade, o que também pode determinar fator limitante para sua administração nos rebanhos.

Os resultados das coproculturas pela técnica de Roberts e O'Sullivan (1950) aplicada nas amostras pré-tratamento indicaram a predominância dos gêneros Trichostrongylus (20\%) e Haemonchus (80\%), que já haviam sido relatados por Ramos et al. (2002) em Santa Catarina.

Chagas et al. (2007) evidenciaram em média $67,7 \%$ de $H$. contortus e $32,3 \%$ de Trichostrongylus sp. em coproculturas ao longo de um ano, demonstrando que são realmente estes os parasitos mais prevalentes em ovinos. No presente estudo, não foram realizadas coproculturas no período pós-tratamentos. 


\section{CONCLUSÃO}

A maioria dos fármacos disponíveis comercialmente não foi eficaz no tratamento antihelmíntico, o que reduz drasticamente o arsenal farmacológico que pode ser empregado e demonstra a necessidade de aprimorar opções de combate aos parasitos que não se baseiem exclusivamente no uso de químicos.

\section{ANTHELMINTIC EFFICACY OF DIFFERENT DRUGS EMPLOYED FOR THE CONTROL OF PARASITES IN SHEEP}

\section{ABSTRACT}

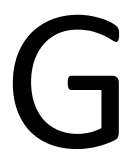
astrointestinal parasitism is the biggest problem faced by sheep farmers. The constant presence of parasites leads producers to use a large number of treatments what causes resistance. The present study aimed to evaluate the efficacy of different chemical groups used for the control ofgastrointestinal nematodes in sheep flocks from Alto Uruguay Catarinense, West of Santa Catarina State. A total of 82 Texel females were used. Eight drugs were tested: closantel, triclorfon, albendazole, disofenol, levamisole, moxidectin, ivermectin, and clorsulon associated with ivermectin. All of them were used through their commercial form, according to the dosage indicated by the Manufacturer Laboratory. The method applied for the evaluation of anthelmintic efficacy was the fecal reduction test, calculated by the percentage of reduction in the number of eggs per gram of faeces (EPG), held from the pre and post-treatment results (seven and fourteen days) and depending on the variation of the EPG of control groups in the same period. Seven of the eight tested drugs, were demonstrated as ineffective for treatments in the studied flock. The percentage of reduction was $54.7 \%$ and $84.3 \%$ for Closantel; $93.1 \%$ and $90.4 \%$ for Triclorfon; $0 \%$ in both analyses for Albendazole; $100 \%$ in both tests for Disofenol; $8,4 \%$ and $0 \%$ for levamisole, and for moxidectin, ivermectin and ivermectin associated with clorsulon the percentages were zero. The results still showed that the parasite genders present in the experiment conduction were Trichostrongylus (20\%) and Haemonchus (80\%). In conclusion, most part of commercially available drugs was not effective, what drastically reduces the pharmacological arsenal that can be used in the West of Santa Catarina State and demonstrates the need of improved options that are not based only in chemicals to combat parasites.

Keywords: Sheep production. Anthelmintic resistance. Helminthosis. 


\section{EFICACIA ANTIHELMÍNTICA DE DIFERENTES FARMACOS UTILIZADOS EN EL CONTROL DE LOS PARÁSITOS EN OVINOS}

\section{RESUMEN}

as parasitosis gastrointestinales son un gran problema en la ovinocultura porque llevan a los productores a utilizar antihelmínticos en exceso, contribuyendo para el desarrollo de resistencia. El presente trabajo tuvo como objetivo evaluar la eficacia antihelmíntica de fármacos para el control de parásitos gastrointestinales en un rebaño de ovejas de la región del Alto Uruguay, al oeste de Santa Catarina. Se utilizaron 82 ovejas de la raza Texel que fueron distribuidas en dos grupos controles y seis grupos para probar los siguientes fármacos: closantel, triclorfón, albendazol, disofenol, levamisol, moxidectina, ivermectina y la ivermectina asociado a clorsulón. Todos fueron utilizados a través de su forma comercial, de acuerdo con la dosis recomendada por el fabricante. La evaluación de la eficacia fue calcula por la porcentaje de reducción del conteo de huevos por gramo de heces (HPG) pre y post- tratamiento (siete y catorce días). Las muestras fecales pre-tratamiento fueron sometidas a coprocultivo para la identificación de los géneros de los parásitos presentes en el rebaño. Siete de los ocho fármacos probados fueron ineficaces para el tratamiento de la helmintiasis. Los porcentajes de reducción fueron $54,7 \%$ y $84,3 \%$ para el closantel, $93,1 \%$ y 90,4\% para el triclorfón, 0\% para ambas contajes del albendazol, 100\% para ambos días el disofenol, $8,4 \%$ y $0 \%$ para el levamisol; y cero para los demás productos (moxidectina, ivermectina e ivermectina asociado a clorsulón). Los géneros de parásitos presentes en el rebaño fueron Trichostrongylus (20\%) y Haemonchus (80\%). Se concluye que la mayoría de los fármacos disponibles a nivel comercial no fueron eficaces en el tratamiento antihelmíntico, lo que demuestra la necesidad de buscar opciones para el combate de los parásitos que no estén basados exclusivamente en el uso de químicos. Estudios complementarios son necesarios para dimensionar la resistencia antihelmíntica en el Oeste de Santa Catarina.

Palabras clave: Ovinocultura. Antihelmíntico. Resistencia. Helmintiasis.

\section{REFERÊNCIAS}

ALMEIDA, M. A. O.; AYRES, M. C. C. Considerações gerais sobre os anti-helmínticos. In: SPINOSA, H. S.; GÓRNIAK, S. L.; BERNARDI, M. M. Farmacologia aplicada à medicina veterinária. Rio de Janeiro: Guanabara Koogan, p. 401-406, 1996.

AMARANTE, A. F. T.; BARBOSA, M. A.; OLIVEIRA, M. R.; et al. Efeito da administração de Oxfendazol, Ivermectina e Levamisole sobre os exames coproparasitológicos de ovinos. Brazilian Journal of Veterinary Research and Animal Science, v. 29, p. 31-38, 1992. 
BOERSEMA, J. H.; PANDEY, V. S. Anthelmintic resistance of trichostrongylids in sheep in the highveld of Zimbabwe. Veterinary Parasitology, v. 68, n. 4, p. 383-388, 1997.

CHAGAS, A. C. de S.; CARVALHO, C. O.; MOLENTO, M. B. Método FAMACHA@: Um recurso para o controle da verminose em ovinos. Circular Técnica Embrapa Pecuária Sudeste, São Carlos, n. 52, 8 p. 2007.

COLES G. C.; ROUSH, R. T. Slowing the spread of anthelmintic-resistant nematodes of sheep and goats in the United Kingdom. Veterinary Record, n. 130, p. 505- 510, 1992.

COLES, G. C. Anthelmintic resistance - looking to the future: a UK perspective. Research Veterinary Science, v. 78, n. 2, p. 99-108, 2005.

CUNHA FILHO, L. F. C.; YAMAMURA, M. H. Resistência a anti-helmínticos em ovinos da região de Tamarana, Paraná, Brasil. Ciência Biológica da Saúde, v. 1, n. 1, p. 31-39, 1999.

ECHEVARRIA, F. A. M.; PINHEIRO, A. C.; CORRÊA, M. B. C. Alternativas para o controle da verminose ovina no Rio Grande do Sul. Comunicado Técnico do Centro Nacional de Pesquisa de Ovinos da EMBRAPA, n. 8, p. 1-6, 1988.

FALBO, M. K.; SOCCOL, V. T.; SANDINI, I. E.; et al. Atividade anti-helmíntica do triclorfon e closantel em cordeiros naturalmente infectados por Haemonchus sp. Ciencia Animal Brasileira, v. 10, n. 3, p. 926-930, 2009.

GORDON, H. McL.; WHITLOCK, H. N. A new technique for counting nematode egg in sheep faeces. Journal of Commonwealth Science and Industrial Organization, v. 12, n. 1, p. 50-52, 1939.

KAPLAN, R. M.; BURKE, J. M.; TERRILL, T. H.; et al. Validation of the FAMACHA eye color chart for detecting clinical anemia in sheep and goats on farms in southern United States.

Veterinary Parasitology, n. 123, p. 105-120, 2004.

NARI, A. H. Towards sustainable parasite control practices in livestock production with emphasis in Latin America. Veterinary Parasitology, v. 180, p. 2-11, 2011.

RAMOS, C. I.; BELLATO, V.; ÁVILA, V. S. de; et al. Resistência de parasitos gastrintestinais de ovinos a alguns anti-helmínticos no estado de Santa Catarina, Brasil. Ciência Rural, v. 32, n. 3, p. 474-477, 2002.

ROBERTS, F. H. S.; O'SULLIVAN, P. J. Methods for eggs counts and larval cultures for Strongyles infesting the gastro-intestinal tract of catlle. Australian Journal of Agriculture Research, v. 1, p. 99-103, 1950. 
SOTOMAIOR, C. S.; CARLI, L. M.; TANGLEICA, L.; et al. Identificação de ovinos e caprinos resistentes e susceptíveis aos helmintos gastrintestinais, Revista Acadêmica, v. 5, n. 4, p. 397-412, 2007.

THOMAZ-SOCCOL, V. T.; SOUZA, F. P.; SOTOMAIOR, C.; et al. Resistance of gastrointestinal nematodes to anthelmintics in sheep (Ovisaries). Brazilian Archives of Biology and Technology, v. 47, p. 41-47, 2004.

TORRES-ACOSTA, J. F. J.; SANDOVAL-CASTRO, C. A.; HOSTE, H.; et al. Nutritional manipulation of sheep and goats for the control of gastrointestinal nematodes under hot humid and subhumid tropical conditions. Small Ruminant Research, n. 103, p. 28-40, 2012.

VERÍSSIMO, C. J.; NICIURA, S. C. M.; ALBERTI, A. L. L.; et al. Multidrug and multispecies resistance in sheep flocks from Sao Paulo state, Brazil. Veterinary Parasitology, v. 187, p. 209-216, 2012.

WOOD, I. B.; AMARAL, N. K.; BAIRDEN, K.; et al. Word Association for the Advancement of Veterinary Parasitology: second edition of guidelines for evaloating the efficacy of anthelmintics in ruminants. Veterinary Parasitology, v. 58, p. 181-213, 1995.

Autor para correspondência:

Plínio Aguiar Oliveira.

Rua Gravataí, no 405, bairro: Laranjal, Pelotas - RS. CEP: 96090-300.

plinio-vet@hotmail.com 